\title{
Modular recursive Green's function method for ballistic quantum transport
}

\author{
Stefan Rotter, Jian-Zhi Tang, Ludger Wirtz, Johannes Trost, and Joachim Burgdörfer \\ Institute for Theoretical Physics, Vienna University of Technology, Wiedner Hauptstrasse 8-10, A-1040 Wien, Austria \\ and Department of Physics, University of Tennessee, Knoxville, Tennessee 37996-1200
}

(Received 12 January 2000)

\begin{abstract}
A modification of the standard recursive Green's function method for quantum transport through microstructures is presented which is based on the decomposition into separable substructures. The Green's functions for these modules are joined by discretized Dyson equations. Nonseparable structures can thereby be calculated with the help of a few recursions with high accuracy. We apply this method to the calculation of ballistic quantum transport through a circular and stadium-shaped quantum dot for high mode numbers to test semiclassical predictions in detail. Among other results we find the breakdown of the semiclassical approximation for long path lengths which is due to the spreading of wave packets in the cavity.
\end{abstract}

\section{INTRODUCTION}

Billiards have long served as prototype systems in the field of classical and quantum chaos. ${ }^{1}$ Investigations on closed billiards have revealed information on the statistics of energy levels ${ }^{2}$ and pronounced enhancements of wave functions near unstable periodic orbits. ${ }^{3}$ Recently, the study of quantum transport through open billiards has received considerable attention due to advances in the fabrication of semiconductor structures which led to the experimental realization of phase coherent scattering devices. ${ }^{4-7}$

The transport properties of these microstructures are dominated by quantum interference in the ballistic regime, resulting in strong conductance fluctuations. The interference pattern depends on the shape of the cavity, the opening of the attached wave guide, and the wavelength of the incident electron. In order to delineate signatures of classically chaotic dynamics in ballistic quantum transport, semiclassical descriptions based on Miller's ${ }^{8}$ and Berry and Mount's ${ }^{9}$ representation of the scattering amplitude have been developed. ${ }^{10-15}$ Since these are asymptotic expansions for small wavelength $\lambda$, classical-quantum correspondence can be explored only for large quantum numbers, i.e., large transverse mode numbers $m$ of the incoming and outgoing wave in the quantum wires with wave numbers

$$
k_{\perp}=\frac{m \pi}{d},
$$

where $d$ is the width of the wire (or lead width). The latter poses however a major challenge, in particular for small lead width $d / D \ll 1$, where $D$ is the typical linear dimension of the quantum dot.

The motion of an electron in a restricted open twodimensional domain appears to be a standard problem of quantum scattering theory. However, for large mode numbers $m \gg 1$, or equivalently, large Fermi wave numbers $k_{F}$, the problem becomes increasingly difficult to handle. Conventional close-coupling schemes are computationally unfeasible, primarily due to the numerical instability introduced by closed channels. ${ }^{16}$ Existing techniques for quantum transport calculations through open quantum dots can be roughly sub- divided into two groups: basis expansion and discretization on a grid. The former approach, as with the mode-matching method ${ }^{17}$ or the expansion in plane and spherical waves, ${ }^{18-20}$ is of high numerical precision for low mode numbers but becomes unstable for high energies and suffers from breakdown of unitarity. Methods employing discretization on a grid, like in the finite element ${ }^{21}$ or recursive Green's function method $^{22-25}$ are stable, preserve unitarity to a high degree, but become numerically inefficient for large $k_{F}$. This is because for small $\lambda$, the density of grid points required to subtend the quantum dot and the attached quantum wires becomes prohibitively large. In the standard recursive Green's function method (RGM) the Green's function is propagated through the scattering region from one transverse strip to the next through repeated solution of a matrix Dyson equation. However, in the semiclassical limit, $m \gg 1, D / d \gg 1$, the high number of large matrix inversions makes this method impractical. A hybrid method combining the RGM with the close-coupling approach has been recently proposed by Zozoulenko et al. ${ }^{26}$

In the following, we propose a variant of the RGM following an earlier suggestion by Sols et al. ${ }^{27,28}$ which appears suitable for extending quantum transport calculations to larger mode numbers towards the semiclassical regime. The underlying idea is to build up two-dimensional nonseparable open quantum dots out of simpler separable substructures. Important examples which we will treat in detail are the circle and the Bunimovich stadium ${ }^{29}$ attached to two quantum wires. The latter geometry can be assembled from two semi-infinite rectangular quantum wires (leads), two semicircles, and one rectangle in the center (Fig. 1). Because of the separability of the Helmholtz equation in each of these substructures, in the following referred to as modules, the wave function and hence the Green's function can be calculated either analytically or by one-dimensional quadrature essentially exactly. For joining the modules we employ the technique of the RGM by representing the coupling between the modules in terms of the hopping matrix elements of a tight-binding Hamiltonian and by solving a matrix Dyson equation at each junction between the modules. The number of recursions required is thereby reduced to the number of modules needed to reconstruct the quantum dot. At junctions 


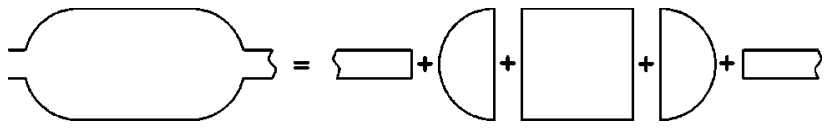

FIG. 1. Schematic illustration of the modular recursive Green's function method as applied to the stadium with opposite leads.

we employ link modules which facilitate the tight-binding coupling between different symmetry-adapted grids in the substructures. Due to the modular structure of the Green's functions employed in our approach, we refer to this extension of the standard RGM as the modular recursive Green's function method (MRGM). This technique is applicable to a large number of open quantum dots that can be constructed from a moderate number of modules for each of which the two-dimensional Helmholtz equation is separable. ${ }^{30}$ The two-dimensional areas of each module have boundaries that correspond to the nodal lines of Cartesian, polar, elliptic, or hyperbolic coordinates. Our method can be extended to a class of locally varying potentials in the dot which preserve the separability of the Schrödinger equation within each module. Furthermore, inclusion of a constant magnetic field is straightforward and will be the subject of a future publication.

We apply the MRGM to a comparative study of transport coefficients for a circle as a prototype of a classically regular system and the Bunimovich stadium as a paradigm for a classically chaotic cavity. Calculations have been performed for mode numbers up to $m=30$ and $m=15$, respectively, to our knowledge the highest ones available to date. We present a detailed comparison with the semiclassical theory of transport through open quantum structures. ${ }^{13-15}$ Among the findings is the identification of signatures of regular and chaotic dynamics in the quantum path-length spectrum and the observation of the breakdown of the semiclassical approximation for very long path lengths.

\section{METHOD}

In this section, we formulate the quantum scattering problem for conductance through ballistic microstructures. Inside the hard-wall boundary of the cavity we assume a constant potential which is infinitely high outside. For the shape of the quantum dot we choose the circle and the stadium as two "typical" examples for regular and chaotic motion (see insets of Figs. 5,6). Two semi-infinite waveguides are attached to the open cavity which are connected to different electrochemical potentials $\left(\mu_{1}, \mu_{2}\right)$ and can thus transport electrons through the microstructure. We choose a narrow opening of the cavity with $d / D=d / \sqrt{A}=0.0935$, where $A=4+\pi$ is the area of the enclosed quantum dot kept fixed for all geometries studied. In the asymptotic regime, i.e., far away from the scattering region, we impose scattering boundary conditions: the wave function can be factorized into a longitudinal propagating part and a transverse standing sine wave, $\chi_{[m]}\left(y_{i}\right)$, with mode number $m$. We choose a local coordinate system in which the variable $x_{i}$ always denotes the longitudinal and $y_{i}$ the transverse direction in the $i$ th lead. For an electron at the Fermi energy $E_{F}$, approaching the cavity in the $m$ th mode, the flux normalized wave functions in the asymptotic regions of lead 1 (entrance lead) and lead 2 (exit lead) are given by

$$
\begin{aligned}
\psi_{[m]}^{1}\left(x_{1}, y_{1}, E_{F}\right)= & \frac{e^{i k_{x_{1}, m} x_{1}}}{\sqrt{k_{x_{1}, m}}} \chi_{[m]}\left(y_{1}\right) \\
& +\sum_{n=1}^{N} r_{n m} \frac{e^{-i k_{x_{1}, n} x_{1}}}{\sqrt{k_{x_{1}, n}}} \chi_{[n]}\left(y_{1}\right), \\
\psi_{[m]}^{2}\left(x_{2}, y_{2}, E_{F}\right)= & \sum_{n=1}^{N} t_{n m} \frac{e^{i k_{x_{2}, n} x_{2}}}{\sqrt{k_{x_{2}, n}}} \chi_{[n]}\left(y_{2}\right) .
\end{aligned}
$$

The $t_{n m}\left(r_{n m}\right)$ are the quantum transmission (reflection) amplitudes from mode $m$ into mode $n$. The index $n$ runs over all possible transverse modes which are determined by the hardwall boundary conditions at $y= \pm d / 2$. In the longitudinal direction the plane wave motion is characterized by the wave vector

$$
k_{x_{i}, n}=\sqrt{k_{F}^{2}-\left(\frac{n \pi}{d}\right)^{2}}
$$

which is real for $n \pi / d<k_{F}$ (open channel) and purely imaginary for $n \pi / d>k_{F}$ (closed channel).

Current conservation is expressed through the unitarity of the $S$ matrix which consists of the transmission and reflection amplitudes

$$
\sum_{n=1}^{N}\left(\left|t_{n m}\right|^{2}+\left|r_{n m}\right|^{2}\right)=1 .
$$

For a phase-coherent system, the transmission through a cavity is directly related to the conductance $g$ by the Landauer formula $^{31}$

$$
g=\frac{2 e^{2}}{h} \sum_{m, n=1}^{N} T_{n m}
$$

where $T_{n m}=\left|t_{n m}\right|^{2}$ is the transmission coefficient. A convenient way of calculating the transmission (reflection) amplitudes is by means of the Green's function which describes the propagation inside the cavity. Projecting this constant energy propagator onto the transverse wave functions in the leads, the transmission amplitudes are given by ${ }^{32,33}$

$$
\begin{aligned}
t_{n m}\left(E_{F}\right)= & -i \hbar \sqrt{v_{x_{2}, n} v_{x_{1}, m}} \\
& \times \int_{-d / 2}^{d / 2} d y_{2} \int_{-d / 2}^{d / 2} d y_{1} \chi_{[n]}^{*}\left(y_{2}\right) \\
& \times G\left(y_{2}, y_{1}, E_{F}\right) \chi_{[m]}\left(y_{1}\right),
\end{aligned}
$$

and a similar relation holds for the reflection amplitudes. The factors $v_{x_{i}, m}=\hbar k_{x_{i}, m} / m_{\text {eff }}$ are the longitudinal velocities of the electron in the $m$ th mode. Atomic units $\left(\hbar=|e|=m_{\text {eff }}\right.$ $=1$ ) will be used from now on, unless explicitly stated otherwise.

\section{A. Tight-binding Green's functions}

Calculating the Green's function for an arbitrarily shaped quantum dot is a nontrivial problem. For many geometries, however, the structure can be decomposed into substructures (modules) that are separable. For these modules, the Green's 
function is either known analytically or can be determined numerically by one-dimensional quadrature. In the following, we will briefly discuss how to construct the Green's function for the separate modules. We then connect these with each other to construct the Green's function of the whole system. Expressing the Green's function at the energy $E$ by means of the eigenvectors $\left|E_{m}\right\rangle$ and the eigenvalues $E_{m}$ of the Hamiltonian $\hat{H}$, we have

$$
G\left(\mathbf{r}, \mathbf{r}^{\prime}, E\right)=\lim _{\epsilon \rightarrow 0^{+}} \sum_{m} \frac{\left\langle\mathbf{r} \mid E_{m}\right\rangle\left\langle E_{m} \mid \mathbf{r}^{\prime}\right\rangle}{E+i \epsilon-E_{m}} .
$$

For simplicity of notation we omit the limit $\left(\lim _{\epsilon \rightarrow 0^{+}}\right)$, imposing outgoing boundary conditions in the following.

We illustrate the calculation of the tight-binding (TB) Green's function for a non-Cartesian grid by the case of polar coordinates [right side of Fig. 2(a)]. Generalizations to other coordinate systems are straightforward. In terms of the separable energy eigenstates $\left|E_{m}\right\rangle=\left|E_{k n}^{\rho}\right\rangle \otimes\left|E_{k}^{\varphi}\right\rangle$, the Green's function can be written as

$$
G\left(\mathbf{r}, \mathbf{r}^{\prime}, E\right)=\sum_{k}\left\langle\varphi \mid E_{k}^{\varphi}\right\rangle\left\langle E_{k}^{\varphi} \mid \varphi^{\prime}\right\rangle \sum_{n} \frac{\left\langle\varrho \mid E_{k n}^{\varrho}\right\rangle\left\langle E_{k n}^{\varrho} \mid \varrho^{\prime}\right\rangle}{E+i \epsilon-E_{k n}} .
$$

The discretization of the Schrödinger equation (and hence of $G)$ in a non-Cartesian grid proceeds through a derivation from a discrete variational principle, using a discretized Lagrangian, to ensure the Hermitian representation of the Hamiltonian on the grid. $^{34}$

This leads to the finite-difference Schrödinger equation

$$
\begin{aligned}
E \Phi_{i, j}= & -\frac{1}{2 \varrho_{i} \Delta \varrho^{2}}\left(\varrho_{i-1 / 2} \Phi_{i-1, j}-2 \varrho_{i} \Phi_{i, j}+\varrho_{i+1 / 2} \Phi_{i+1, j}\right) \\
& -\frac{1}{2 \varrho_{i}^{2} \Delta \varphi^{2}}\left(\Phi_{i, j-1}-2 \Phi_{i, j}+\Phi_{i, j+1}\right)
\end{aligned}
$$

The wave function $\Phi_{i, j}$ in Eq. (2.9) is defined on radial gridpoints $\varrho_{i}=|i-1 / 2| \Delta \varrho$ and on angular sites $\varphi_{j}=j \Delta \varphi$, where $\Delta \varrho$ and $\Delta \varphi$ are the spacings in the radial and angular coordinates. Mapping this expression onto a cylindrical tightbinding Hamiltonian of the form

$$
\begin{aligned}
\hat{H}^{\mathrm{tb}}= & \sum_{i=1}^{N^{\varrho}} \sum_{j=1}^{N^{\varphi}}\left[\left(\varepsilon_{i}^{\varrho}+\varepsilon_{j}^{\varphi}\right)|i, j\rangle\left\langle i, j\left|+V_{i, i-1}^{\varrho}\right| i, j\right\rangle\langle i-1, j|\right. \\
& +V_{i, i+1}^{\varrho}|i, j\rangle\left\langle i+1, j\left|+V_{j, j-1}^{\varphi}\right| i, j\right\rangle\langle i, j-1| \\
& \left.+V_{j, j+1}^{\varphi}|i, j\rangle\langle i, j+1|\right],
\end{aligned}
$$

we can directly read off the hopping matrix elements and the site energies for the cylindrical grid

$$
\begin{gathered}
V_{i, i \pm 1}^{\varrho}=-\frac{\varrho_{i \pm 1 / 2}}{2 \varrho_{i} \Delta \varrho^{2}}, \quad \varepsilon_{i}^{\varrho}=-V_{i, i+1}^{\varrho}-V_{i, i-1}^{\varrho}=\frac{1}{\Delta \varrho^{2}}, \\
V_{j, j \pm 1}^{\varphi}=-\frac{1}{2 \varrho_{i}^{2} \Delta \varphi^{2}}, \quad \varepsilon_{j}^{\varphi}=-V_{j, j+1}^{\varphi}-V_{j, j-1}^{\varphi}=\frac{1}{\varrho_{i}^{2} \Delta \varphi^{2}} .
\end{gathered}
$$

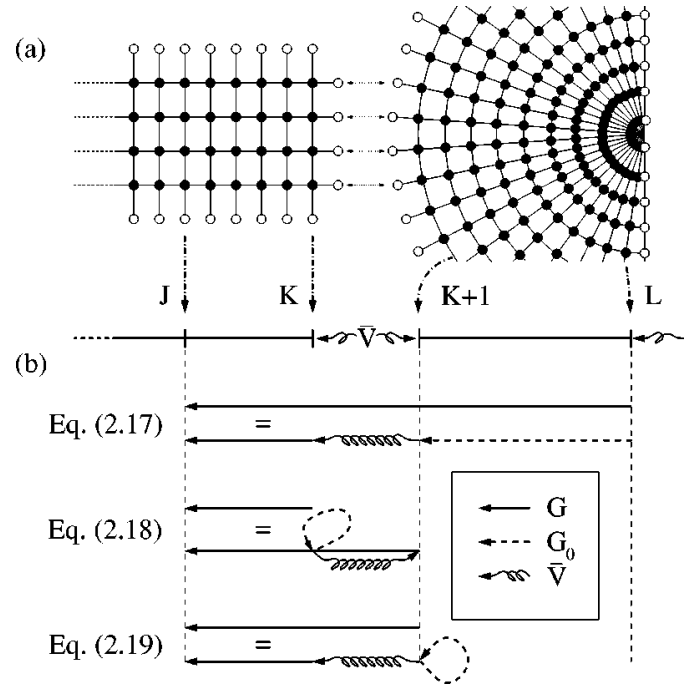

FIG. 2. (a) Diagrammatic representation of the joining of modules by recursion. As an example we show the connection of a lead to a semicircle. The open disks indicate the hard wall boundary conditions. (b) Diagrammatic illustration of the corresponding Dyson equation.

For both the circle and the circle segment, we can incorporate the Dirichlet boundary conditions into the Hamiltonian (2.10) through the requirement $V_{N{ }^{\varrho}, N^{\varrho}+1}^{\varrho}=0$. For the segment we have in addition $V_{1,0}^{\varphi}=V_{N^{\varphi}, N^{\varphi}+1}^{\varphi}=0$. After separation of the radial and angular part $\Phi_{i, j}=R_{i} \cdot \Lambda_{j}$ and the substitution $g_{i}=R_{i} \sqrt{\varrho_{i}}$, Eq. (2.9) is transformed into a tridiagonal and symmetric eigenproblem of the size $N^{\varrho}$ $\times N^{\varrho}$, which is computationally easy to handle,

$$
\begin{aligned}
E(\xi) g_{[\xi], i}= & -\frac{\xi}{\varrho_{i}^{2}} g_{[\xi], i}+\frac{1}{2 \Delta \varrho^{2}}\left(-\frac{\varrho_{i-1 / 2}}{\sqrt{\varrho_{i-1}} \sqrt{\varrho_{i}}}\right. \\
& \left.\times g_{[\xi], i-1}+2 g_{[\xi], i}-\frac{\varrho_{i+1 / 2}}{\sqrt{\varrho_{i}} \sqrt{\varrho_{i+1}}} g_{[\xi], i+1}\right) .
\end{aligned}
$$

The separation constant, the effective squared angular momentum of the discretized problem is defined through $\xi$ $=\left(1 / 2 \Delta \varphi^{2}\right)\left[\left(\Lambda_{j-1}-2 \Lambda_{j}+\Lambda_{j+1}\right) / \Lambda_{j}\right]$. For a full circle with $N^{\varphi}$ angular grid spacings the angular eigenfunctions and eigenvalues read

$$
\Lambda_{[k], j}=\left[\frac{1}{N^{\varphi} \Delta \varphi}\right]^{1 / 2} e^{i k 2 \pi j / N^{\varphi}}, \quad \xi_{k}=\frac{1}{\Delta \varphi^{2}}\left[\cos \left(\frac{2 k \pi}{N^{\varphi}}\right)-1\right],
$$

while for a segment of a circle with $N^{\varphi}$ angular sites we have

$$
\begin{gathered}
\Lambda_{[k], j}=\left[\frac{2}{\left(N^{\varphi}+1\right) \Delta \varphi}\right]^{1 / 2} \sin \left[k \pi j /\left(N^{\varphi}+1\right)\right], \\
\xi_{k}=\frac{1}{\Delta \varphi^{2}}\left[\cos \left(\frac{k \pi}{N^{\varphi}+1}\right)-1\right] .
\end{gathered}
$$

The radial eigenvectors $g_{[\xi, n]}, n \in\left\{1, \ldots, N^{\varrho}\right\}$ are subject to Dirichlet boundary conditions $\left(g_{[\xi], N} \varrho_{+1}=0\right)$ and normal- 
ized by weighting them with the size of the unit cells of the cylindrical grid $\left(\Delta_{R}=\varrho_{i} \Delta \varrho \Delta \varphi\right)$,

$$
\delta_{n, n^{\prime}}=\sum_{i=1}^{N^{\varrho}}\left(R_{[\xi, n], i} R_{\left[\xi, n^{\prime}\right], i}\right) \varrho_{i} \Delta \varrho=\sum_{i=1}^{N^{\varrho}}\left(g_{[\xi, n], i} g_{\left[\xi, n^{\prime}\right], i}\right) \Delta \varrho .
$$

With these expressions we can solve the matrix eigenproblem (2.13). For each $k \in\left\{1, \ldots, N^{\varphi}\right\}$ we obtain $N^{\varrho}$ different eigenvalues $E_{\xi_{k}, n}$ and eigenvectors $g_{\left[\xi_{k}, n\right]}=R_{\left[\xi_{k}, n\right]} \sqrt{\varrho_{i}}$. The results for $\Lambda_{[k]}, R_{\left[\xi_{k}, n\right]}$, and $E_{\xi_{k}, n}$ are then inserted in Eq. (2.8) to calculate the Green's function for modules which are separable in polar coordinates. Analogous expressions can be determined for other curvilinear grids.

Joining different modules proceeds by solving a Dyson equation which we illustrate by the example depicted in Fig. 2. Consider the Green's function describing the propagation from the radial slice $L$ to the Cartesian slice $J$,

$$
G_{L, J}=\underbrace{G_{L, J}^{0}}_{=0}+G_{L, K+1}^{0} \bar{V}_{K+1, K} G_{K, J}
$$

with $\bar{V}_{K+1, K}=V_{K+1, K} \Delta_{R}$. In Eq. (2.17), $G_{L, J}^{0}$ stands for the Green's function of the disconnected modules while $G$ is the Green's function of the connected, enlarged system. The indices $K$ and $K+1$ refer to the slices of grid points on both sides of the border between the modules to be connected. Note that the hopping matrix is weighted with the area of the unit cell at the junction of two tight-binding grids. Each multiplication in Eq. (2.17) stands for a matrix multiplication where the dimension is given by the number of sites within the slice. In order to close the system of matrix equations, two more Dyson equations are needed [see Fig. 2(b)]

$$
G_{K, J}=G_{K, J}^{0}+G_{K, K}^{0} \bar{V}_{K, K+1} G_{K+1, J},
$$

and finally

$$
G_{K+1, J}=\underbrace{G_{K+1, J}^{0}}_{=\mathbf{0}}+G_{K+1, K+1}^{0} \bar{V}_{K+1, K} G_{K, J} .
$$

From the coupled equations above and the boundary conditions at the borders we obtain

$$
\begin{aligned}
G_{L, J}= & G_{L, K+1}^{0} \bar{V}_{K+1, K}\left(1-G_{K, K}^{0} \bar{V}_{K, K+1}\right. \\
& \left.\times G_{K+1, K+1}^{0} \bar{V}_{K+1, K}\right)^{-1} G_{K, J}^{0} .
\end{aligned}
$$

This recursion method has the advantage that it involves only one inversion of a matrix for connecting two entire modules. Furthermore, for connections performed at lead mouths, the dimension of the involved matrices along the border is small due to the small number of grid points required.

In the case of attaching a lead to a cylindrical module we can employ an additional approximation. Under the assumption, that the opening of the lead is small compared to the radius of the module, we can approximate the arc of the circle at the lead mouth to be a straight line [see Fig. 2(a)]. This approximation is valid as long as the wavelength of the electron is large compared to the error in spacing induced by

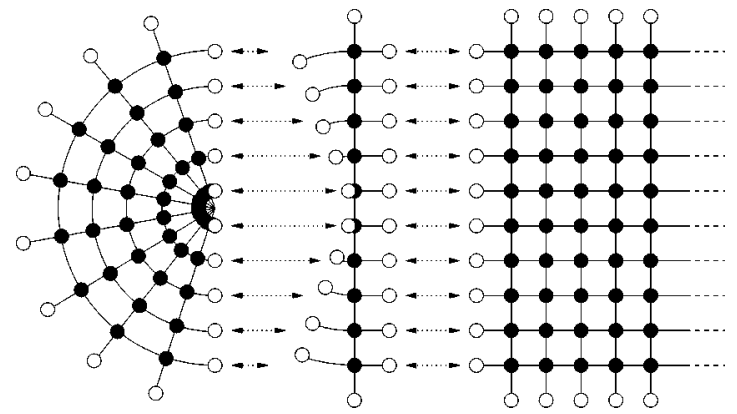

FIG. 3. Link module ("double wedge") for connecting a cylindrical with a Cartesian grid.

this assumption. For keeping the Hamiltonian at this junction Hermitian, we have to make sure that the Cartesian hopping potential in propagating direction is equal to the radial component of the cylindrical hopping potential $\left(V^{x}=-1 / 2 \Delta x^{2}\right.$ $\left.=V_{N e, N e+1}^{\varrho}\right)$. We must also require that the Cartesian and the cylindrical unit cells at the junction have the same size $\left(\Delta x \Delta y=\varrho_{N} \Delta \varrho \Delta \varphi\right)$.

\section{B. Link modules}

The nontrivial key feature of the present method is the connection between modules of different symmetries which cannot be dealt with by the approximation mentioned above. This task is performed by "link modules" that are plugged in between the modules of different grid structure (Fig. 3). We illustrate this method explicitly for the link between a Cartesian and a cylindrical module. Two of such links are required for the construction of a stadium which results from joining two semicircles (cylindrical module with $\varphi=\pi$ ) and a rectangle (Cartesian module). This link module is referred to in the following as a double wedge. Its construction in terms of a tight-binding Hamiltonian proceeds as follows: the double wedge is half a cylindrical wedge and half a Cartesian strip (see Fig. 3), consisting of three neighboring chains. One corresponds to the "last" radial chain of the cylindrical grid, the other is the "first" Cartesian chain along the $y$ direction and the third one in the middle is the "hybrid" strip $(\Delta y=\Delta \varrho)$. The boundary conditions for the wedge are such that the wave function vanishes at the outer two chains. The finite-difference Schrödinger equation for the double wedge reads

$$
\begin{aligned}
E \frac{\Delta \varrho}{2}\left(\left|\varrho_{i}\right| \Delta \varphi+\Delta x\right) \Phi_{i}= & \Phi_{i}\left[\frac{\Delta \varrho \Delta \varphi}{2}\left(\frac{\varrho_{i}}{\Delta \varrho^{2}}+\frac{1}{\varrho_{i} \Delta \varphi^{2}}\right)\right. \\
& \left.+\frac{\Delta \varrho \Delta x}{2}\left(\frac{1}{\Delta y^{2}}+\frac{1}{\Delta x^{2}}\right)\right] \\
& -\frac{1}{2} \Phi_{i+1}\left[\frac{\Delta \varphi \varrho_{i+1 / 2}}{2 \Delta \varrho}+\frac{\Delta \varrho \Delta x}{2 \Delta y^{2}}\right] \\
& -\frac{1}{2} \Phi_{i-1}\left[\frac{\Delta \varphi \varrho_{i-1 / 2}}{2 \Delta \varrho}+\frac{\Delta \varrho \Delta x}{2 \Delta y^{2}}\right],
\end{aligned}
$$


where the cell area of the hybrid strip is $\Delta_{R}$ $=(\Delta \varrho / 2)\left(\left|\varrho_{i}\right| \Delta \varphi+\Delta x\right)$.

The wave function is again zero on the boundary $\left(\Phi_{-N} \varrho=\Phi_{N \varrho+1}=0\right)$. Writing Eq. (2.21) in matrix notation, we obtain a generalized eigenproblem of the form $E \hat{B}|\Phi\rangle$ $=\hat{H}|\Phi\rangle$. The matrix $\hat{H}$ corresponds to the (symmetric) Hamiltonian and the diagonal matrix $\hat{B}$ determines the orthonormalization of the generalized eigenvectors of $\hat{H}$,

$$
\begin{gathered}
\langle\Phi|\hat{B}| \Phi\rangle=\mathbf{I} \Leftrightarrow \\
\delta_{m, m^{\prime}}=\sum_{i=-N^{\varrho}+1}^{N^{\varrho}} \Phi_{[m], i} \Phi_{\left[m^{\prime}\right], i} \frac{\Delta \varrho}{2}\left(\varrho_{i} \Delta \varphi+\Delta x\right) .
\end{gathered}
$$

With the above expressions the Green's function for the double wedge $G_{\mathrm{DW}}^{0}$ can be calculated in the same way as for a one-dimensional finite chain.

It is instructive to visualize the coupling of the double wedge to the cylindrical and Cartesian modules with the help of the one-dimensional (1D) problem of joining a semicircle along the $\varphi$ direction, at fixed $\varrho_{i}$, with a straight line along the $x$ direction, at fixed $y$. With the abbreviations

$$
\begin{aligned}
& V^{\varphi}=-\frac{1}{2 \varrho_{i}^{2} \Delta \varphi^{2}} \hat{=} \nu, \quad-2 V^{\varphi} \hat{=} \varepsilon, \\
& V^{x}=-\frac{1}{2 \Delta x^{2}} \hat{=} \nu^{\prime}, \quad-2 V^{x} \hat{=} \varepsilon^{\prime},
\end{aligned}
$$

the 1D TB-Hamiltonian for this composition is given by the following symmetric tridiagonal matrix

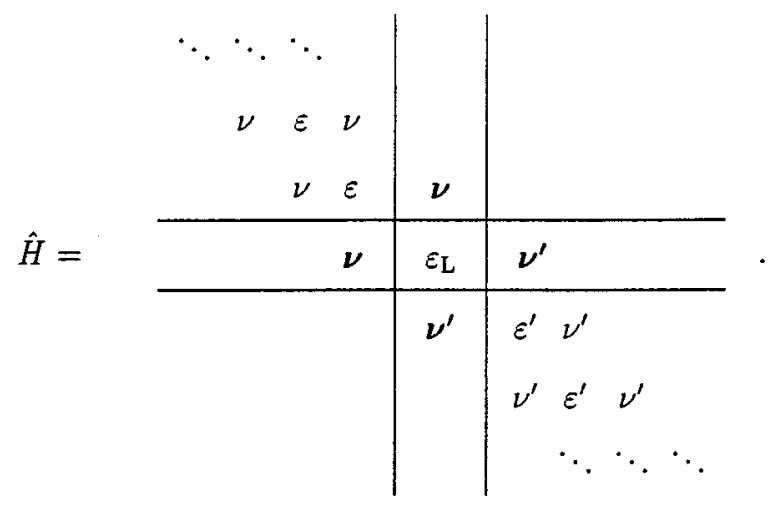

We have boldfaced those hopping matrix elements which would be zero in the case of disconnected modules. Within this 1D Hamiltonian, the link consists of a single site energy $\varepsilon_{L}=\left[1 /\left(2 \varrho_{i}^{2} \Delta \varphi^{2}\right)+1 /\left(2 \Delta x^{2}\right)\right]$. For the full (2D) problem, the double wedge consists of all sites along the wall between the Cartesian and the cylindrical grid with $i=-N_{\varrho}$ $+1, \ldots, N_{\varrho}$ (note that $N_{y}=2 N_{\varrho}$ ). In the recursion the link module is "attached" to the Cartesian (cylindrical) grid by means of the hopping potential $\bar{V}^{x}\left(\bar{V}^{\varphi}\right)$, respectively.

\section{Remarks on the efficiency of the MRGM}

With the help of the Green's function for the lead $G_{\mathrm{L}}^{0}$, the semicircle $G_{\mathrm{SC}}^{0}$, the double wedge $G_{\mathrm{DW}}^{0}$, and the rectangle $G_{\mathrm{RA}}^{0}$, the Green's function $G$ for the composite structure (the stadium) can be determined. This requires a total of six recursive solutions of the Dyson equation corresponding to the number of border slices in between the modules. (This number can be lowered by symmetry considerations.) By comparison, in the standard RGM the Green's function is calculated recursively at every new slice in propagating direction. This amounts to a large number of recursions, i.e., matrix inversions, of the order of $n_{\|} \gg 1$. Each of these recursions requires the inversion of a matrix whose size is determined by the number of grid points in transverse directions which is of the order of $n_{\perp} \gg 1$. Therefore, for high mode numbers and short wavelengths, a large number of grid points and of inversions are needed, which renders this method eventually impractical. In the MRGM described above, the number of inversions is only given by the number of modules needed to build the structure, independent of the wavelength. Moreover, the size of the matrices involved in joining the leads with the structure is modest, such that the number of inversions of large matrices in the present case of the stadium is reduced to 4 . In the regular structures such as the circle, no inversion of large matrices is required at all. The present method can be straightforwardly extended to other geometries and structures with the only limitation that these can be built up from a modest number of separable modules. Furthermore, inclusion of a homogeneous magnetic field is possible for structures that can be decomposed into modules for which the separability is preserved upon a suitable gauge transformation. Another advantage of the MRGM is that changes in position and direction of the lead can be done without any major effort. There are, however, several limitations to this approach. (1) When the scattering device can not be built up from separable modules our approach fails. (2) Random potentials for disordered systems that break the symmetry of the module cannot be treated. However, any smooth potential including those depending on random variables can be included as long as they preserve separability of the substructure. (3) The MRGM is not suited for yielding the wave function in the scattering region, although this is possible in principle. $^{24}$

\section{NUMERICAL RESULTS}

In this section we will present the transmission and reflection amplitudes and probabilities for the circle and the Bunimovich stadium as prototypes for regular and chaotic quantum dots. The emphasis is laid on large values of $k_{F}$, or equivalently, on high mode numbers which were not easily accessible by previous calculation methods and which permit detailed comparison with semiclassical predictions. We will present results for different lead geometries that place different weights on the role of short and long paths.

\section{A. Numerical tests}

Apart from convergence checks as a function of the number of grid points, conservation of unitarity provides a sensitive measure for the numerical accuracy. All data presented 


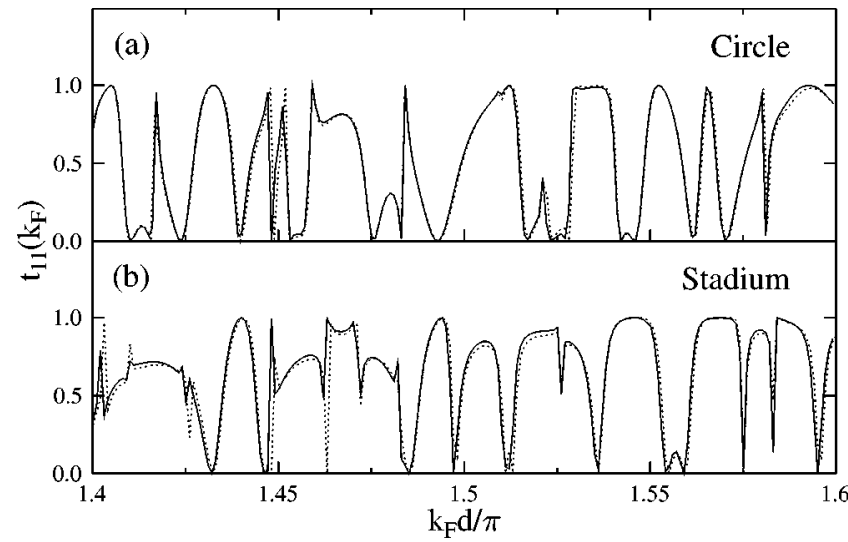

FIG. 4. Comparison between the present MRGM (solid line) and the mode-matching method (Ref. 18) (dotted line) for the firstmode transmission probability $\left[T_{11}=\left|t_{11}\left(k_{F}\right)\right|^{2}\right]$ in a small window of $k_{F}$ : (a) circle with opposite leads, (b) stadium with opposite leads.

in the following display a violation of unitarity of less than $10^{-4}$. Within the accuracy of our calculations the error of the transport coefficients (as a result of a variation of the grid size) stays below $1 \%$. In addition, for low values of $k_{F}$, a direct comparison for $T_{n m}\left(k_{F}\right)$ and $R_{n m}\left(k_{F}\right)$ with previous methods can be made. As an example, Fig. 4 displays the comparison for the transmission coefficient in the first propagating mode, $T_{11}\left(k_{F}\right)$ with the wave function matching method of Nakamura and Ishio ${ }^{18}$ for (a) the circle and (b) the stadium. In both cases the rapid fluctuations in the transmission coefficient agree very well, despite the fact that for the stadium the violation of unitarity in the wave function matching method is already of the order of $20 \%$.

\section{B. Transmission coefficients and conductance fluctuations}

The $k_{F}$ dependence of the total transmission is displayed in Fig. 5 for the circle and in Fig. 6 for the stadium. We calculated 200 points per mode number, up to $m_{\max }=30$ for the circle and $m_{\max }=15$ for the stadium. To our knowledge, this calculation encompasses the largest range of wavenumbers available at present. In both figures, part (a) displays the data for the geometry with opposite leads and part (b) for perpendicular leads. In each case, the staircase function represents the sum of transmission and reflection, which, according to the unitarity condition, is equal to the number of open channels in the quantum wires. The classical transmission and reflection are linear functions whose slope is determined by the fraction of trajectories that leave the cavity via exit or entrance lead. In order to illustrate the changes in the mean values of conductance $\langle T\rangle$ and resistance $\langle R\rangle$ we display smoothed curves averaged over the rapid conductance fluctuations. For better visibility, the exact quantum results are only shown for conductance. Strong irregular fluctuations are visible which are a realization of Ericson fluctuations. These are expected when the resonance overlap criterion $\langle\Gamma\rangle /\langle\Delta E\rangle \geqslant 1$ is satisfied, where $\langle\Gamma\rangle$ is the mean width of the resonance in the quantum dot and $\langle\Delta E\rangle$ is the average level spacing between resonances. The latter can be estimated from the Weyl formula for the semiclassical level density $^{1} \varrho$

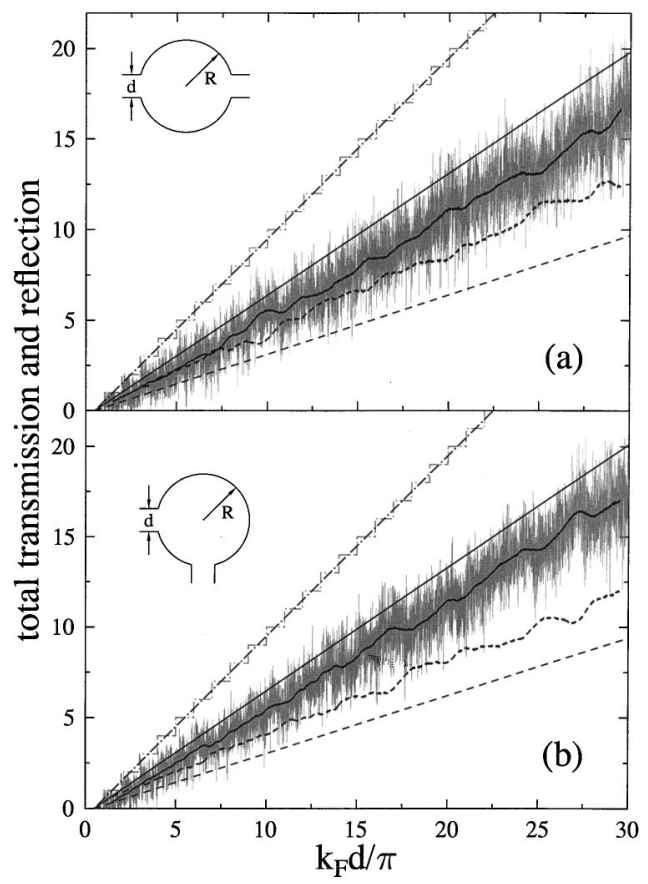

FIG. 5. The total transmission probability $T^{\mathrm{qm}}=\Sigma_{n, m}\left|t_{n m}\right|^{2}$ (gray solid line) as a function of the wave vector $k_{F}$. The staircase function, $T^{\mathrm{qm}}+R^{\mathrm{qm}}$ (gray dash-dotted line), represents the quantum current conservation condition. The straight solid (dashed) line is the classical transmission $T^{\mathrm{cl}}$ (reflection $R^{\mathrm{cl}}$ ) with the corresponding total classical current as a dash-dotted straight line. The continuous black (dashed) curve is the mean quantum transmission (reflection) as an average over $\Delta k_{F}=\pi / d$ : (a) for the circle with opposite leads, (b) for circle with perpendicular leads.

$$
\langle\Delta E\rangle=\varrho^{-1} \approx 2 \pi / A,
$$

where $A$ is the area of the quantum dot. For chaotic systems, $\langle\Gamma\rangle$ can be assessed from the "universal" classical dwell time distribution $P(t)=e^{-\Gamma t}$. The magnitude of $\Gamma$ is deter-

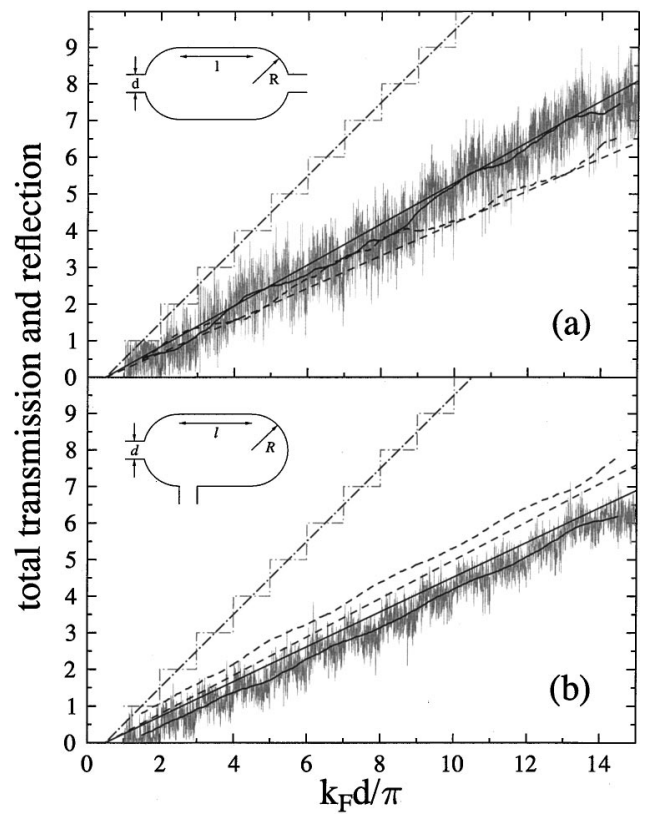

FIG. 6. As in Fig. 5 but for (a) the stadium with opposite leads and (b) the stadium with perpendicular leads $l / R=2$. 
mined by the path length $(L)$ distribution, which displays an exponential decay, however strongly modulated by nonuniversal classical fluctuations. We analyze this in detail below [see Eq. (3.8)]. Our calculations give

$$
\langle\Gamma\rangle=\langle L\rangle^{-1} v_{F} \simeq 0.0223 \times v_{F} \simeq 0.0223 \mathrm{~m} \pi / d,
$$

thus leading to

$$
\frac{\langle\Gamma\rangle}{\langle\Delta E\rangle} \simeq 0.0112 m\left(\frac{A}{d}\right) .
$$

In scaled units the geometry of the cavity is determined by the area $A=4+\pi$. For both, the circle and the stadium, the lead width is given by $d=0.0935 \sqrt{A}$. Consequently

$$
\frac{\langle\Gamma\rangle}{\langle\Delta E\rangle} \simeq 0.119 \sqrt{A} m \simeq 0.319 m .
$$

The regime of Ericson fluctuations is reached for moderately high mode numbers $(m \geq 3)$. Indeed, for the test case of $m$ $=1$ (Fig. 4) several isolated resonances can still be identified, but with increasing $m$ this is no longer feasible (not shown). For regular structures, the universal estimate for the path length and dwell time distributions does not apply. Nevertheless, from the numerically determined mean value of $\langle L\rangle$ a similar estimate for the regime of overlapping resonances can be made.

Despite the fact that the present calculation of conductance fluctuations extends to higher values of $k$ than previous investigations, we find that the semiclassical calculation of $T(k)$ and $R(k)$, performed in the primitive semiclassical approximation (PSC) as well as by including diffractive corrections ${ }^{13,14}$ fails to quantitatively account for the fluctuations. Moreover, unitarity is strongly violated..$^{15}$ Even for large $k_{F}$, no improvement in the absolute amount of violation was discernible. A more instructive quantitative comparison with semiclassical calculations employing the pathlength spectrum will be presented below.

Another characteristic feature of the quantum mechanical results is the deviation of the mean values of transmission and reflection from the corresponding classical curves for the stadium with perpendicular leads and for the circle with both lead orientations. These deviations are often referred to as weak localization (WL). ${ }^{10}$ The most prominent contribution to the WL effect for the structures with perpendicular leads is "coherent backscattering" (CB): ${ }^{12}$ time reversal symmetry of reflected paths leads to constructive interference and thereby to an average enhancement of the diagonal reflection coefficients $\left|r_{n n}\left(k_{F}\right)\right|^{2}$. For the stadium with opposite leads, no systematic WL effect is observed. This is due to the horizontal symmetry axis of the structure. Every path has a reflection symmetric counterpart leading to constructive interference in both diagonal and off-diagonal parts of transmission and reflection. (For the diagonal part of the reflection, this symmetry coincides with time-reversal symmetry, and therefore the enhancement due to constructive interference coincides with the CB effect.) Since the stadium is a chaotic structure where long trajectories are randomly distributed, the enhancement effects for reflection and transmission cancel each other out and no overall WL effect is visible in Fig. 6(a), in agreement with the findings of Ref. 35.
For the circle with opposite leads [Fig. 5(a)] which has the same discrete symmetry properties as the stadium in Fig. 6(a), one might also expect a cancellation of enhancement effects. However, this structure is not chaotic and transport is determined by nonrandomized path bundles. Therefore the enhancement effects do not necessarily cancel out. Indeed, we observe a strong enhancement of the quantum reflection compared to the classical value. This finding is in disagreement with the semiclassical investigation of Ref. 12, where it was observed that $\mathrm{CB}$ for the diagonal part of the reflection was cancelled out by an "anti-WL" effect for the offdiagonal contributions to the reflection. However, in Ref. 12 a much wider lead width is used and, possibly, the amount of cancellation depends on the lead width, which in turn influences the weight of longer trajectories. We also find that the offset of the quantum from the classical transmission (reflection) increases approximately linearly as a function of $k$ for the integrable cavities whereas no systematic trend was observed in the chaotic case.

As a measure for the strength of the interference in quantum transport, we display in Fig. 7 the mean of the conductance fluctuations $\sigma(T)=\left\langle(T-\langle T\rangle)^{2}\right\rangle^{1 / 2}$. Random matrix theory (RMT) predicts that in chaotic structures without a discrete symmetry, the mean fluctuation approaches the values $1 / \sqrt{8}$ for high mode numbers. ${ }^{36}$ Indeed, the mean fluctuation for the stadium with perpendicular leads follows this prediction (lower dotted curve). The mean for the stadium with opposite leads approaches a value close to $1 / \sqrt{2}$ (upper dotted curve) as predicted for chaotic structures with horizontal and vertical reflection symmetry. ${ }^{35}$ The deviation from the RMT prediction is possibly due to the fact that our geometries allow direct (nonchaotic) trajectories connecting entrance and exit lead. The analysis for the two circular geometries shows strongly enhanced fluctuations relative to the RMT prediction, because transport is mediated by nonrandomized path bundles in these regular structures. Furthermore we find that the fluctuations are growing with increasing wave number $k$. This is in agreement with recent semiclassical work on conductance through another integrable cavity, i.e., the rectangular billiard ${ }^{37}$ (see also Ref. $10)$.

\section{Path-length spectra}

In order to analyze the influence of regular and chaotic classical dynamics in more detail and to perform a meaningful comparison with semiclassical theory, we analyze the conductance and resistance in terms of the path length distribution contributing to transport. The method consists of a Fourier transformation of the transmission (or reflection) amplitude $^{38}$

$$
\tilde{t}_{n m}(L)=\int_{k_{\min }}^{k_{\max }} d k_{F} t_{n m}\left(k_{F}\right) \exp \left(-i k_{F} L\right) .
$$

In the following, the variable $L$, conjugate to $k_{F}$, will be referred to as the path length. This identification is unambiguous within the semiclassical description where the amplitude $t_{\mathrm{nm}}\left(k_{F}\right)$ is written in terms of a coherent superposition of amplitudes of paths $q$ with a given length $L_{q}$, 


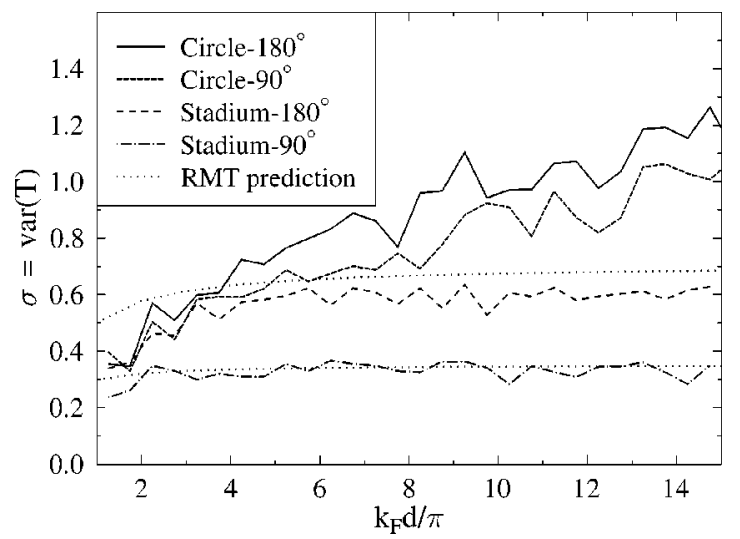

FIG. 7. Mean of conductance fluctuations $\sigma=\left\langle(T-\langle T\rangle)^{2}\right\rangle^{1 / 2}$ for four different scattering geometries as a function of $k_{F}$. The variance $\sigma$ is obtained by averaging over $100 k_{F}$ values in the total transmission amplitude within the interval $\left[k_{F} \pm \Delta\right]$. For the stadium geometry with perpendicular leads, $\sigma$ agrees very well with the results of random matrix theory (Ref. 36) (lower dotted curve). For the stadium geometry with opposite leads, the RMT prediction for cavities with fourfold symmetry (Ref. 35) (upper dotted curve) is quite close to our results.

$$
t_{n m}\left(k_{F}\right)=\frac{2 \pi}{(2 \pi i)^{3 / 2}} \sum_{q}\left|D_{\mathrm{q}}\right|^{1 / 2} e^{i k_{F} L_{\mathrm{q}}-i(\pi / 2) \mu_{\mathrm{q}}} .
$$

We denote the weighting factor for each path (or bundle of paths) by $D_{\mathrm{q}}$ and the Maslov index by $\mu_{\mathrm{q}}$. (For details, see Refs. 13-15).

Studying the power spectrum derived from the quantum transmission amplitudes $\left|\widetilde{t}_{n m}(L)\right|^{2}$, we can gain information about the underlying classical dynamics of the scattering process. ${ }^{13-15,38}$ High resolution of the $L$ spectra requires data for a large interval of wave numbers $\left[k_{\min }, k_{\max }\right]$ such that the resulting width of structures in $L$ is not Fourier transform limited but reveals information about the intrinsic peak width due to the spread of $L$ within the bundles of trajectories. In all the data presented in this paper, the transform limited length width is $\Delta L_{\mathrm{FT}}=\pi /\left(k_{\max }-k_{\text {min }}\right) \approx 0.054$ for the circle $(\approx 0.11$ for the stadium $)$. This results in path-length spectra of unprecedented resolution, even for large values of $L$. Figures 8 and 10 present the path-length spectra for the circle and the stadium (both lead geometries). The path length spectrum for regular structures (Fig. 8) consists of a sequence of isolated sharp peaks which can be unambiguously associated with individual bundles of trajectories. Most prominent is the family of "asterisk" trajectories (shown as insets) with a pattern of increasing number of bounces at the wall accompanied by a revolution within the structure before exiting. The near perfect agreement between the present quantum calculation and the semiclassical approximation for the position of the peaks reveals the close classical-quantum correspondence for this system. It illustrates that the pathlength spectrum can provide detailed insights into the quantum dynamics. There are only few deviations where nonclassical paths significantly contribute to the conductance, one of which is highlighted by an arrow [see Fig. 8 (a)]. The peak at $L \approx 9$ originates from a "ghost" path and illustrates the importance of diffraction effects. The contributing trajectories correspond to three straight-line traversals of the circle in-

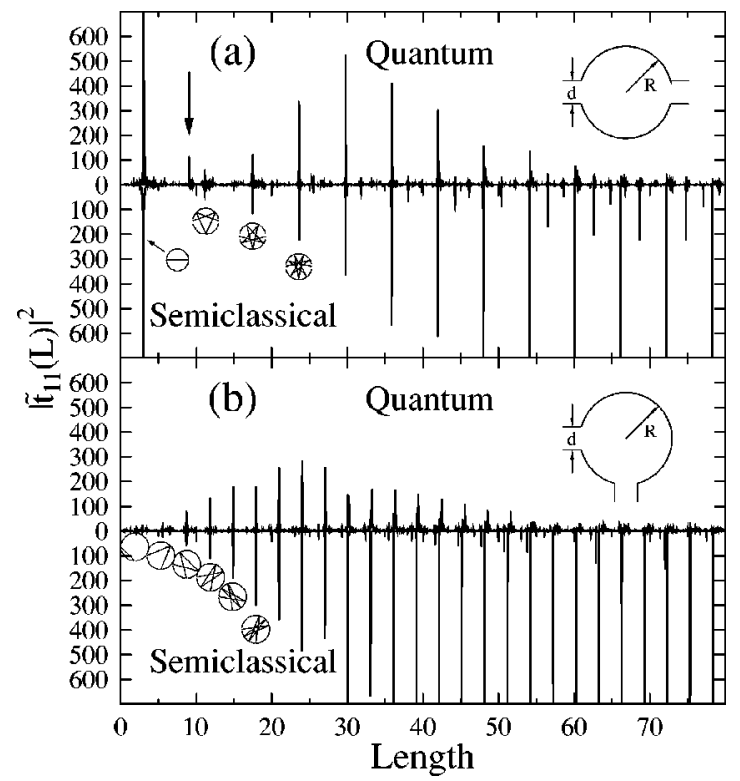

FIG. 8. Power spectrum $\left|\tilde{t}_{11}(L)\right|^{2}$ of the transmission amplitude for a finite window of $k_{F}, 1 \leqslant k_{F} \leqslant 30$, in units of $\pi / d$. Cavity area $A=4+\pi$ and lead width $d=0.0935 \times \sqrt{A}$ : (a) for the circle billiard with opposite leads (b) for the circle with perpendicular leads (the height of some peaks in the spectrum is off the scale). Individual peaks can be associated with classical bouncing patterns, which are shown as insets.

cluding two diffractive reflections from the lead mouths before exiting on the third "try."' Ghost paths are absent in the PSC approximation but can be incorporated within the framework of diffractive corrections. ${ }^{13}$ In addition to such individual nonclassical contributions, the present numerical results reveal a systematic and increasing failure of the semiclassical approximation at large path length $L$ (see Fig. 8). While the peak height, i.e., the weight of a given path bundle within the spectrum, is represented quite well by semiclassical theory for small to moderate $L$, the semiclassical approximation (SCA) overestimates the contribution for long paths. One consequence of the failure is the breakdown of the SCA for the description of the conductance fluctuations $T_{n m}\left(k_{F}\right)$. The conductance amplitude in $k$ space $t_{n m}\left(k_{F}\right)$ consists of a coherent superposition of all path amplitudes. The failure to properly represent the path amplitudes for large $L$ leads, among other things, to a gross overestimate of the conductance fluctuation and hence to the violation of unitarity.

It is instructive to investigate the origin of the failure of the SCA. In Fig. 9 we give the ratio of the semiclassical to the quantum peak height as a function of the path length $L$ where we have grouped trajectories according to their injection angle into the billiard. Small injection angles $\theta$ (measured relative to the $x_{1}$ axis in the lead) correspond to the trajectories with low angular momenta $l$ which come close to the center of the circle. Large angles $\theta$ correspond to trajectories with large angular momenta that stay mostly at the outer circumference. For both groups of trajectories the ratio of peak heights increases rapidly with $L\left(\sim L^{4}\right)$, alluding to the influence of the spreading of the quantal wavepacket beyond the classical spread after many bounces. ${ }^{14}$ Surprisingly, the lower $l$ is the stronger the spreading is enhanced. This indicates that the effect of wave packet spreading is more 


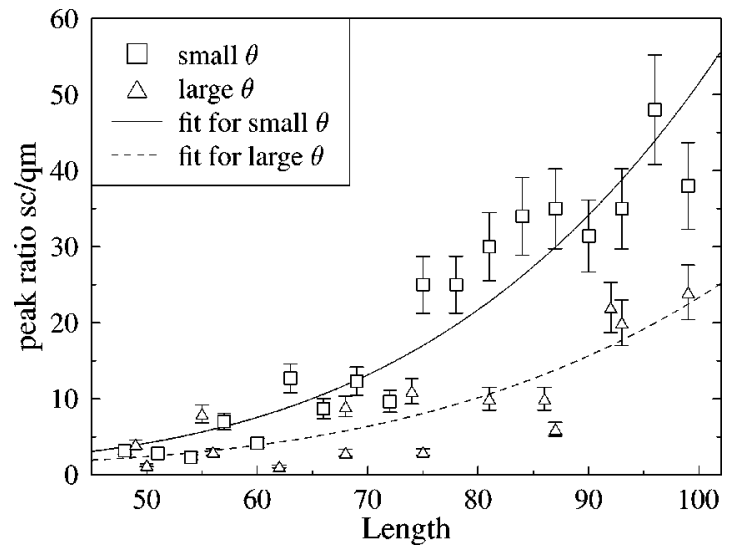

FIG. 9. Ratio of semiclassical to quantum peak heights in the $\left|\tilde{t}_{11}(L)\right|^{2}$ and $\left|\tilde{r}_{11}(L)\right|^{2}$ power spectra for the two circle geometries, as a function of the path length. The ratio is larger for peaks with a small incident angle $\theta$ (measured relative to the $x_{1}$ axis). The solid lines are fits to the values for small and large incident angle respectively (fit function: $1+a \times \mathrm{L}^{4}$ ).

pronounced at traversals of inner classical turning points than at bounces with the cavity walls. ${ }^{39}$

These results raise interesting conceptual questions: classical chaotic dynamics leaves clear, "universal," imprints only for long trajectories. Therefore, signatures of chaotic dynamics in quantum transport, if existent, should originate from trajectories for large $L$. However, the present findings suggest that precisely in this region of the path-length spectrum, the classical-quantum correspondence breaks down. The question is then posed: how can quantum transport carry information of classical chaos?

A partial resolution to this puzzle is contained in the pathlength spectrum for the chaotic stadium with different lead orientations (see Fig. 10). These spectra differ qualitatively and quantitatively from the spectra for regular systems (Fig. 8). Rather than sharp discrete peaks, the spectrum contains a strongly fluctuating quasicontinuum indicating a large number of overlapping path bundles. More precisely, the intrinsic width $\Delta L$ of the bundles is larger than the spacing between different bundles. This can be viewed as the analog to Ericson fluctuations but in $L$ rather than in $k$ space. Notice that this observation is not an artifact produced by the width of the Fourier transform interval, since the corresponding transform limited width $\Delta L_{\mathrm{FT}}$ is generally smaller than the average intrinsic width. The important point we note is that these differences appear already at modest path lengths where the classical-quantum correspondence still holds and the semiclassical approximation yields good agreement.

The appearance of a quasicontinuum is related to the exponential proliferation of orbits in chaotic structures which is, in turn, closely related to the exponential divergence of nearby trajectories described by a positive Lyapunov exponent. Figure 11 displays the classical deflection function $D_{q}(L)$ for a trajectory $q$ as a function of the length $L$, in the stadium with perpendicular leads. Both the entrance position $y_{1}$ as well as the entrance angle $\sin \theta_{1}$ were chosen randomly. Since the deflection function $D_{q}$ describing the stability of the trajectory $q$ is inversely proportional to the divergence of neighboring paths, the absolute magnitude of the deflection factor $\left|D_{q}\right|$ roughly falls off as $\exp \left(-\lambda L_{q}\right)$, where

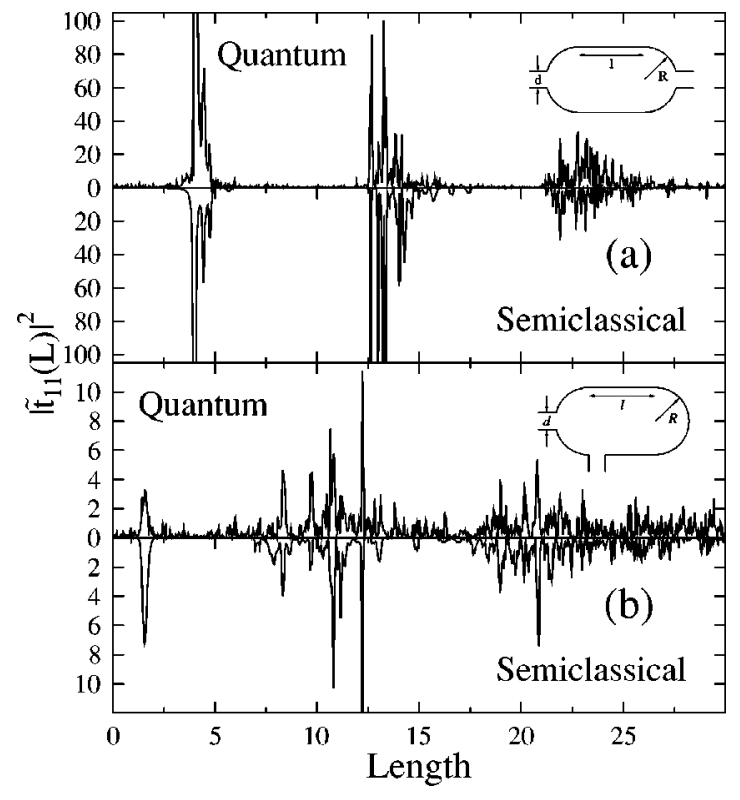

FIG. 10. Power spectrum $\left|\tilde{t}_{11}(L)\right|^{2}$ of the transmission amplitude for a finite window of $k_{F}, 1 \leqslant k_{F} \leqslant 15$, in units of $\pi / d$. Cavity area $A=4+\pi$ and lead width $d=0.0935 \times \sqrt{A}$ : (a) for the stadium billiard with opposite leads (b) for the stadium with perpendicular leads. Note the different vertical scales in (a) and (b).

$\lambda$ is the Lyapunov exponent. In the present context of direct significance is the clustering and the exponential proliferation of trajectories at moderate path length. The first accumulation at $L_{q} \approx 2$ clearly corresponds to a bundle of paths which directly connects the entrance to the exit lead without " "exploring" the cavity. At $L_{q} \approx 10$ we find a large number of trajectories which have crossed the vertical symmetry axis of the (closed) stadium exactly two times before exiting. Every further accumulation of paths (at $L_{q} \approx 20,30, \ldots$ ) implies two more traversals of this axis. Also the other accumulations in Fig. 11 can be explained by characteristic bouncing patterns. The exponential proliferation of the number of orbits is proportional to $D_{q}^{-1} \propto \exp (\lambda L)$ since the divergence is a direct measure of the partitioning and folding of the phase space. Due to the high density of the trajectories, both the clustering and the exponential proliferation can be clearly seen in the path length spectrum of the quantum conductance as a quasicontinuous distribution (Fig. 10). Each broad peak at $L \simeq n \times 10(n=1,2, \ldots)$ is associated with $2 n$ traversals of the vertical symmetry line of the closed stadium. Equally, for the stadium with opposite leads, broad peaks appear at distances corresponding to two traversals of the vertical symmetry axis. Since these signatures appear at moderate path lengths, where the classical-quantum correspondence is well satisfied, the quasicontinuous path-length spectrum, modulated by the clustering of trajectories, is a generic feature of chaotic transport through quantum dots. One salient feature of the characterization of transport properties in terms of a path-length spectrum is that the presence of direct or "nonchaotic" paths can be clearly separated and does not influence the analysis of generic properties of chaotic dynamics. The latter are indeed structurally identical for the stadium with opposite and perpendicular leads while the weight of direct paths is vastly different for both geometries. No such 


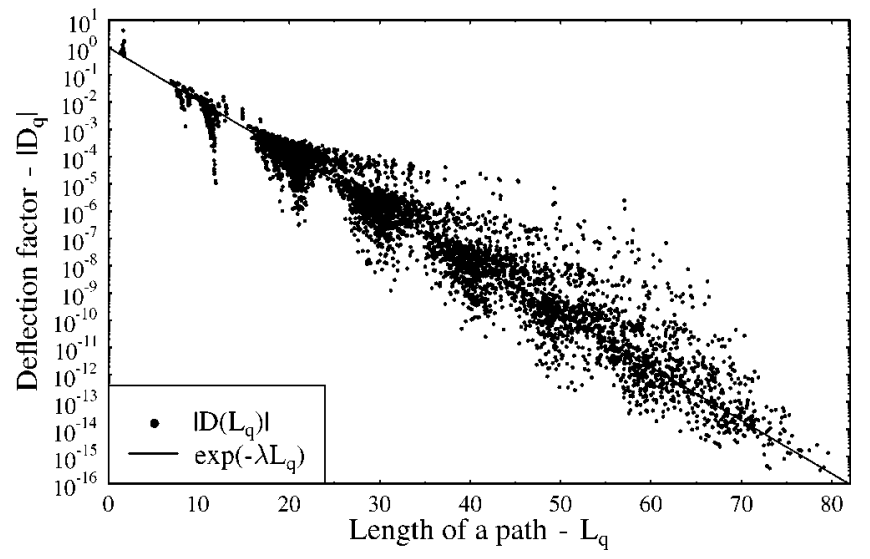

FIG. 11. The absolute value of the deflection factor $\left|D_{\mathrm{q}}(L)\right|$, as a function of the trajectory length, in the stadium billiard with perpendicular leads. Each point represents a randomly chosen trajectory, propagating from the entrance to the exit lead.

clear separation can be achieved for the $k$-dependent conductance $T_{n m}(k)$ due to the presence of quantum interference between direct (nongeneric) paths and multibounce paths. The latter leads for the stadium with opposite leads to nonuniversal mean fluctuations comparable to regular structures. Moreover, the quasicontinuous, randomly fluctuating $L$ spectrum can be associated with universal conductance fluctuations (Fig. 6) while a discrete $L$ spectrum gives rise to larger, nonuniversal fluctuations (Fig. 5).

The degree of classical-quantum correspondence can be further highlighted when one analyzes the total path-length spectrum, summed over all modes

$$
T^{\mathrm{qm}}(L)=\sum_{n, m}\left|\tilde{t}_{n m}(L)\right|^{2} .
$$

In the classical limit, $n, m \gg 1, T^{\mathrm{qm}}(L)$ should tend to the classical path-length spectrum $P^{\mathrm{cl}}(L)$, i.e., the path length distribution averaged over all injection and summed over all ejection angles. Due to the fact that the present calculation extends to high mode numbers, we can test this conjecture directly. Figure 12 displays the remarkable agreement between $P^{\mathrm{cl}}(L)$ and $T^{\mathrm{qm}}(L)$ for path lengths up to $L \simeq 100$. The direct paths, as well as the modulation due to the clustering of trajectories, are clearly visible. Moreover, for long path length the modulation decays, leaving us with the universal exponential path length distribution ${ }^{11}$

$$
P^{\mathrm{cl}}(L)=\kappa e^{-\kappa L}
$$

with a decay constant $\kappa=2 d / A \pi \simeq 0.0223$. The convergence of the quantum path length distribution towards the classical limit may be surprising at first glance considering the strong discrepancies for individual mode-selected paths (see Fig. 9).

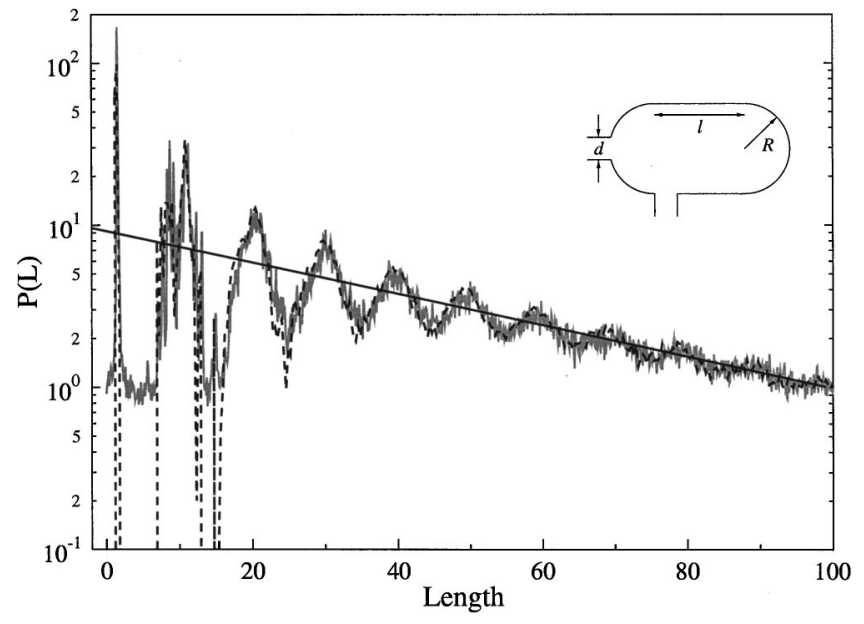

FIG. 12. Comparison between the classical path-length distribution $P^{\mathrm{cl}}(L)$ (black dashed line) and the total power spectrum $T^{\mathrm{qm}}(L)=\sum_{n, m}^{10}\left|\tilde{t}_{n, m}(L)\right|^{2},\left(10 \leqslant k_{F} d / \pi \leqslant 15\right)$ (gray solid line) for the stadium billiard with perpendicular leads. $(A=4+\pi, d=0.0935$ $\times \sqrt{A})$. The straight solid line represents the exponential decay with an exponent $\kappa \simeq 0.0223$ (classical escape rate) (Ref. 11).

It appears that the redistribution of amplitude among different paths for an exponentially growing number of trajectories is due to wave packet spreading and cancels out in the sum over all modes.

\section{SUMMARY}

We have presented a method of calculating transport coefficients through open quantum dots, the modular recursive Green's function method (MRGM) which is a variant of a standard recursive Green's function method. Its key feature is the decomposition of nonseparable structures into separable substructures which are joined by recursive solutions of the Dyson equation. With this method we were able to determine the conductance and resistance amplitudes for high mode numbers, thereby approaching the semiclassical regime. We have analyzed the path-length spectrum of the conductance amplitude and have found the breakdown of the semiclassical approximation for large path length. Furthermore we have identified unambigous signatures of chaotic transport in terms of a quasicontinuous path-length spectrum as opposed to a discrete spectrum for regular structures. These signatures remain unaffected from the presence of direct paths, the breakdown of the semiclassical approximation for long paths and the contributions of nonclassical paths. Work on the inclusion of a magnetic field for the analysis of magnetoconductance is in progress.

\section{ACKNOWLEDGMENTS}

We thank Hiromu Ishio for putting his computer codes at our disposal. This work was supported by the NSF and FWF. 
${ }^{1}$ M. C. Gutzwiller, Chaos in Classical and Quantum Mechanics (Springer Verlag, New York, 1991), and references therein.

${ }^{2}$ G. Bohigas, M. Giannoni, and C. Schmit, Phys. Rev. Lett. 52, 1 (1984).

${ }^{3}$ E. J. Heller, Phys. Rev. Lett. 53, 1515 (1984).

${ }^{4}$ See, e.g., Quantum Transport in Ultrasmall Devices, edited by D. Ferry, H. Grubin, C. Jacobini, and A.-P. Jauho, Vol. 342 of NATO Advanced Studies Institute Series B: Physics (Plenum, New York, 1995).

${ }^{5}$ See, e.g., Nanostructure Physics and Fabrication, edited by M. A. Reed and W. P. Kirk (Academic, New York, 1989).

${ }^{6}$ C. M. Marcus, A. J. Rimberg, R. M. Westervelt, P. F. Hopkins, and A. C. Gossard, Phys. Rev. Lett. 69, 506 (1992); C. M. Marcus, R. M. Westervelt, P. F. Hopkins, and A. C. Gossard, Surf. Sci. 305, 480 (1994).

${ }^{7}$ M. W. Keller, O. Millo, A. Mittal, D. E. Prober, and R. N. Sacks, Surf. Sci. 305, 501 (1994).

${ }^{8}$ W. Miller, Adv. Chem. Phys. 25, 69 (1974).

${ }^{9}$ M. Berry and K. Mount, Rep. Prog. Phys. 35, 315 (1972).

${ }^{10}$ H. U. Baranger, R. A. Jalabert, and A. D. Stone, Chaos 3, 665 (1993).

${ }^{11}$ W. A. Lin, J. B. Delos, and R. V. Jensen, Chaos 3, 655 (1993).

${ }^{12}$ W. A. Lin and R. V. Jensen, Phys. Rev. B 53, 3638 (1996).

${ }^{13}$ C. D. Schwieters, J. A. Alford, and J. B. Delos, Phys. Rev. B 54, 10652 (1996).

${ }^{14}$ L. Wirtz, J.-Z. Tang, and J. Burgdörfer, Phys. Rev. B 56, 7589 (1997).

${ }^{15}$ L. Wirtz, J.-Z. Tang, and J. Burgdörfer, Phys. Rev. B 59, 2956 (1999)

${ }^{16}$ P. Burke and W. Robb, Adv. At. Mol. Phys. 11, 143 (1975); B. R. Johnson, J. Comput. Phys. 13, 445 (1973).

${ }^{17}$ R. L. Schult, H. W. Wyld, and R. G. Ravenhall, Phys. Rev. B 41, 12760 (1990).
${ }^{18}$ K. Nakamura and H. Ishio, J. Phys. Soc. Jpn. 61, 3939 (1992).

${ }^{19}$ E. Doron, U. Smilansky, and A. Frenkel, Physica D 50, 367 (1991).

${ }^{20}$ X. Yang, H. Ishio, and J. Burgdörfer, Phys. Rev. B 52, 8219 (1995).

${ }^{21}$ Y. Yang, J. Wang, and H. Guo, Phys. Rev. B 49, 1928 (1994).

${ }^{22}$ P. A. Lee and D. S. Fisher, Phys. Rev. Lett. 47, 882 (1981).

${ }^{23}$ D. J. Thouless and S. Kirkpatrick, J. Phys. C 14, 235 (1981).

${ }^{24}$ H. U. Baranger, D. P. DiVincenzo, R. A. Jalabert, and A. D. Stone, Phys. Rev. B 44, 10637 (1991).

${ }^{25}$ R. Akis, D. K. Ferry, and J. P. Bird, Phys. Rev. Lett. 79, 123 (1997).

${ }^{26}$ I. V. Zozoulenko, F. A. Maaø, and E. H. Hauge, Phys. Rev. B 53, 7975 (1996).

${ }^{27}$ F. Sols, M. Macucci, U. Ravaioli, and K. Hess, J. Appl. Phys. 66, 3892 (1989).

${ }^{28}$ F. Sols, Ann. Phys. (N.Y.) 214, 386 (1991), and references therein.

${ }^{29}$ L. A. Bunimovich, Funct. Anal. Appl. 8, 254 (1974).

${ }^{30}$ P. M. Morse and H. Feshbach, Methods of Theoretical Physics (McGraw Hill, New York 1953), p. 494 ff.

${ }^{31}$ R. Landauer, IBM J. Res. Dev. 1, 223 (1957).

${ }^{32}$ A. D. Stone and A. Szafer, IBM J. Res. Dev. 32, 384 (1988).

${ }^{33}$ H. U. Baranger and A. D. Stone, Phys. Rev. B 40, 8169 (1989).

${ }^{34}$ S. E. Koonin and D. C. Meredith, Computational Physics (Addison Wesley, Reading, MA, 1990), Chap. 6.

${ }^{35}$ H. U. Baranger and P. A. Mello, Phys. Rev. B 54, R14 297 (1996).

${ }^{36}$ H. U. Baranger and P. A. Mello, Phys. Rev. Lett. 73, 142 (1994).

${ }^{37}$ P. Pichaureau and R. A. Jalabert, Eur. Phys. J. B 9, 299 (1999).

${ }^{38}$ H. Ishio and J. Burgdörfer, Phys. Rev. B 51, 2013 (1995).

${ }^{39}$ H. Friedrich and J. Trost, Phys. Rev. Lett. 76, 4869 (1996). 